\title{
TAMIZAJE FITOQUÍMICO Y CÁLCULO DE RENDIMIENTO DE SAPOGENINAS ESTEROIDALES DE TRES PROCEDENCIAS DE SOLANUM QUITOENSE UAR. SEPTENTRIONALE "NARANJILLO"
}

Palabras clave: Solanaceae, sapogeninas alcaloidales, sapogeninas estereoidales, hormonas, corticoide, elucidación, espectroscópicas.

Key Words: Solanaceae, alkaloidal sapogenins, steroidal sapogenins, hormones, corticoids, elucidation, spectroscopic.

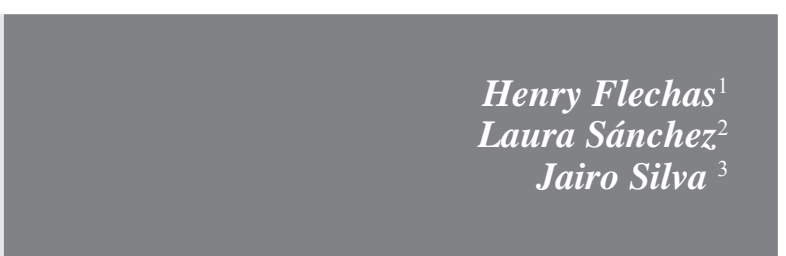

ABSTRACT

This research includes the study of aerial parts (fruits) of lulo de monte (Solanum quitoense var. Septentrionale). The main objective of this research was to determine the secondary metabolites and especially the presence and quantity of alkaloidal saponins, which are common in the Solanaceae family. These substances are used as precursors for the manufacture of several steroid-type drugs, hormones and anti-inflammatories.

The study was conducted with species of three different origins and three different maturing stages gathered in the department of Cundinamarca, Colombia between 2.400 and 2.600 meters above sea level during the period from March to June. Sapogenins were extracted, isolated and purified through various techniques used for this purpose. The presence of saponins was proved through physical and chemical analysis, and the structural elucidation through NMR and IR spectroscopic techniques.

The presence of hecogenin in green fruits from the three sources was determined. This molecular structure corresponds to a non-steroidal sapogenin reported in this species.

Se determinó la presencia de hecogenina en frutos verdes de las tres procedencias. Esta estructura molecular corresponde a una sapogenina esteroidal no reportada en esta especie. 


\section{INTRODUCCIÓN}

Las sapogeninas esteroidales obtenidas en general a partir de especies del género Solanum son de importancia farmacéutica, porque se utilizan como materia prima para la producción de medicamentos esteroidales (Fontan 1973). Dentro de este género existen plantas nativas y poco estudiadas, las cuales constituyen no sólo una fuente de sapogeninas esteroidales sino también una alternativa para recuperar zonas aptas para su cultivo. Éstas son de crecimiento rápido, producen todo el año, no requieren suelos ricos ni cuidados especiales y presentan una amplia distribución en todo tipo de climas. Debido al gran consumo a nivel mundial, se hace necesaria la obtención de esteroides por métodos más eficientes, económicos y a partir de fuentes de origen vegetal, cumpliendo con las nuevas tendencias ambientales para el uso de recursos locales y plantas nativas.

Los extractos naturales estandarizados ricos en sapogeninas tienen muchas aplicaciones en varios sectores de la industria. En farmacéutica los mayores usos de estos productos están en la síntesis de hormonas, drogas para el tratamiento contra el cáncer y el alzheimer, control del colesterol e investigación de vacunas humanas (herpes y HIV), entre otros. En cosmética se emplean para preparar champús, acondicionadores, cremas para cuidar la piel, pomadas hidratantes, tónicos para estimular el crecimiento del cabello, tratamientos para la seborrea y jabones. Las sapogeninas en la industria nutracéutica han demostrado ser útiles como potenciadoras del sistema inmunológico humano, además tienen propiedades antioxidantes y surfactantes, sirven como preservativos solubles en agua y espumantes para licores. En veterinaria las sapogeninas se emplean como adyuvante para vacunas animales, para potenciar el sistema inmunológico de los animales sin tener el efecto nocivo de los antibióticos, reducen los niveles de amoniaco en la cría de cerdos, así como también reducen el colesterol en huevos de gallina y carne de bovinos; también se emplean para el aumento de la rata de crecimiento del cultivo de peces y crustáceos (Ibáñez 1981). El mercado de extractos vegetales medicinales se calcula en 16.5 millardos, así mismo, las ventas de estas medicinas vienen creciendo aproximadamente un $25 \%$ en los últimos años, lo que muestra que el material médico vegetal es utilizado cada vez más por un gran número de industrias. Los recursos naturales renovables en Colombia no han sido explotados comercialmente en forma adecuada y los recursos vegetales como fuente de metabolitos secundarios han sido estudiados tímidamente. Con este trabajo se pretende continuar la investigación en el aspecto fitoquímico y crear las bases para el mercadeo y desarrollo de un proyecto productivo de sapogeninas a partir de la biodiversidad colombiana en pro de la salud humana.

La importancia de este trabajo radicó en contribuir como estudio exploratorio de la especie, para lo cual se determinaron los principales componentes químicos y se obtuvieron las sapogeninas esteroidales por medio de una metodología eficiente y rentable -con rendimientos superiores a los reportados para la familia solanaceae, que oscilan entre 3.31 y $6.96 \%$ en peso, a partir del crudo y entre 0.073 y $0.102 \%$ a partir de sapogeninas en alto estado de pureza-, con el fin de ofrecer mayor cantidad de ellas, obtenidas a partir de productos naturales.

\section{METODOLOGÍA}

La recolección del material vegetal se realizó en Granada, Nazareth y el Alto de San Miguel, Cundinamarca, a una altura de 2.400-2.600 msnm, con temperaturas entre 18 y $23^{\circ} \mathrm{C}$, y se escogieron frutos de árboles en sus diferentes estados de maduración: verdes, $3 / 4$ de maduración y maduros.

Al material frutal seco se le determinó la cantidad de humedad, de acuerdo al método (Fanor 2001), después se realizó el tamizaje fitoquímico para los tres estados de maduración (Ciulei 1982), lixiviándolos a través de etanol, éter y agua (Figura 1). Las fracciones se concentraron a $200 \mathrm{ml}$, el extracto etéreo se dividió en dos alícuotas de $20 \mathrm{y}$ 180, las cuales corresponden a la fracción A y B. A se sometió a pruebas para triterpenos y esteróles, ácidos grasos y carotenoides, y B para alcaloides, carotenoides, flavonoides, cumarinas y emodinas. El extracto etanólico se concentró a $200 \mathrm{ml}$, se dividió en dos alícuotas de $100 \mathrm{ml}$, se concentró una 


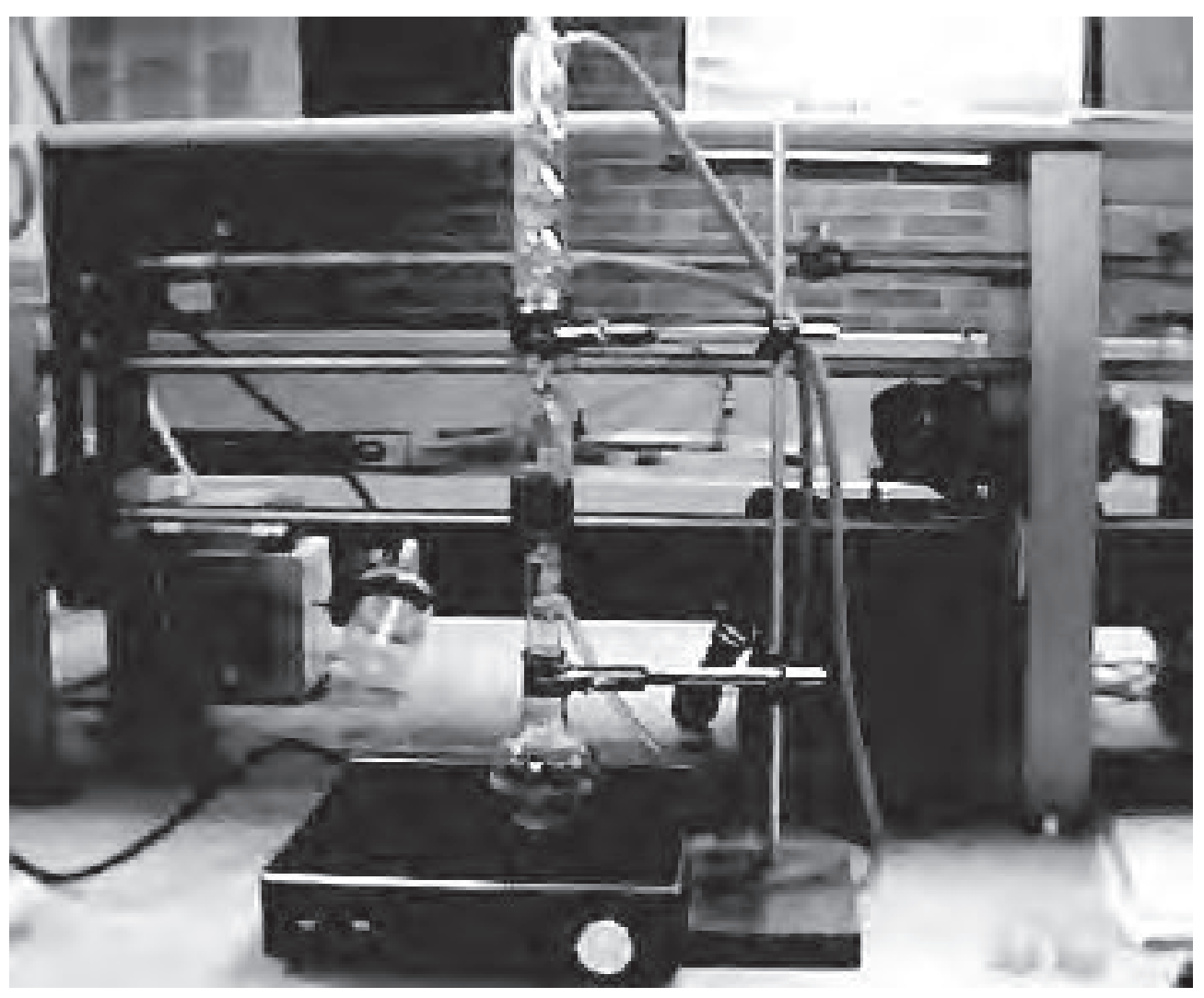

Figura 1. Montaje de extracción continua con etanol de frutos de Solanum quitoense var. septentrionale.

de ellas a $30 \mathrm{ml}$ (fracción E1) y la otra a $50 \mathrm{ml}$ (fracción E2). A E1 se le hicieron pruebas para taninos catéquicos y compuestos reductores y a E2 cumarinas, antocianinas y antracenósidos. Para el extracto acuoso se dividió en dos alícuotas de 100 $\mathrm{ml}$. Una de las alícuotas se utilizó en las pruebas de almidón, taninos catéquicos y taninos gálicos y la otra para las pruebas de polisacáridos y saponinas.

La extracción de sapogeninas se realizó de acuerdo al protocolo (Schafer et al. 2006) utilizado para varias especies del género Solanum. Se lixivió el material en etanol frío ( 3 días) y en caliente (6 horas), y luego se reunieron los extractos.

Obtención del crudo de saponinas. Los extractos A se pasaron por carbón activado, tierra de diatomeas y bentonita (1:2:3, Figura 2). Se fraccionaron con butanol hasta máximo agotamiento, obteniendo seis fracciones. Se unieron las fracciones (B1, B2 y B3) y se dejó en reposo aproximadamente por una hora y se formaron unos cristales que tenían forma de aguja. Éstos fueron filtrados para realizar pruebas de saponinas. El extracto B1 se concentró y se observaron los mismos cristales que los obtenidos inicialmente de coloración parda. A los cristales y crudo de saponinas se le realizaron pruebas cualitativas para dicho grupo fitoquímico. Se realizó lo mismo con B2 y B3.

Los extractos B1, B2 y B3 se hidrolizaron con $\mathrm{H}_{2} \mathrm{SO}_{4}$ diluido, en vez de HCL, debido a que algunos autores lo recomiendan (Sanabria 1983). Se observaron dos fases, una de color oscuro y la otra líquida de color amarillo. Esta última con olor dulce característico de los azúcares. La mezcla se dejó en reposo por doce horas en un embudo de decantación. Para comprobar la hidrólisis completa de los extractos, se realizaron pruebas a las dos fases. Se llevó a sequedad y se fraccionó con cloroformo analítico a reflujo durante una hora, hasta máximo agotamiento. Se observaron varios cristales al concentrar los extractos $\mathrm{C} 1, \mathrm{C} 2$ y $\mathrm{C} 3$. 
Los cristales de $\mathrm{C} 1, \mathrm{C} 2$ y $\mathrm{C} 3$ fueron analizados por $\mathrm{CCD}$, utilizando como fase estacionaria sílica gel $60 \mathrm{H}$ y como fase móvil cloroformo: etanol (0.5:12.5) revelándose con vainillina-ácido sulfúrico, teniendo como patrón hecogenina para confirmar la presencia de sapogeninas en dicho sólido. Lo anterior se complementó con pruebas cualitativas para la determinación de sapogeninas.

Métodos espectroscópicos de las fracciones C1A, C2A y C3A. Los espectros IR de las fracciones se determinaron en un equipo ATI Mattson Génesis Serie FTIR. Las muestras se solubilizaron en cloroformo. Luego se realizaron pruebas espectrométricas de resonancia magnética nuclear, para lo cual se usaron $10 \mathrm{mg}$ y $4 \mathrm{mg}$ de cada una de las muestras en $0.7 \mathrm{ml}$ de cloroformo deuterado. Sobre esta solución se tomaron los espectros unidimensionales $\left({ }^{1} \mathrm{H},{ }^{13} \mathrm{C}\right.$, DEPT 135$)$ y luego los espectros bidimensionales HMQC, HMBC y COSY. Para realizar el análisis se utilizó un equipo Bruker 400 MHz.

\section{RESULTADOS}

\section{GRUPOS FITOQUÍMICOS \\ PRESENTES EN LA ESPECIE POR ESTADO DE MADURACIÓN}

Después de realizar el tamizaje fitoquímico se observó, para las tres procedencias, una incidencia de los grupos de interés (sapogeninas esteroidales) en los frutos verdes. En cuanto al resto de especies químicas se observaron resultados positivos para triterpenos, esteroles y saponinas en CCD y en placa gelatina-sangre, donde se hallaron zonas hemolizadas en los tres estados de maduración.

\section{SAPOGENINAS ESTEROIDALES}

A PARTIR DEL TAMIZAJE FITOQUÍMICO PRELIMINAR

Se utilizaron los frutos verdes de cada procedencia, porque fue donde se encontraron evidencias cualitativas de la presencia de dichas especies químicas.

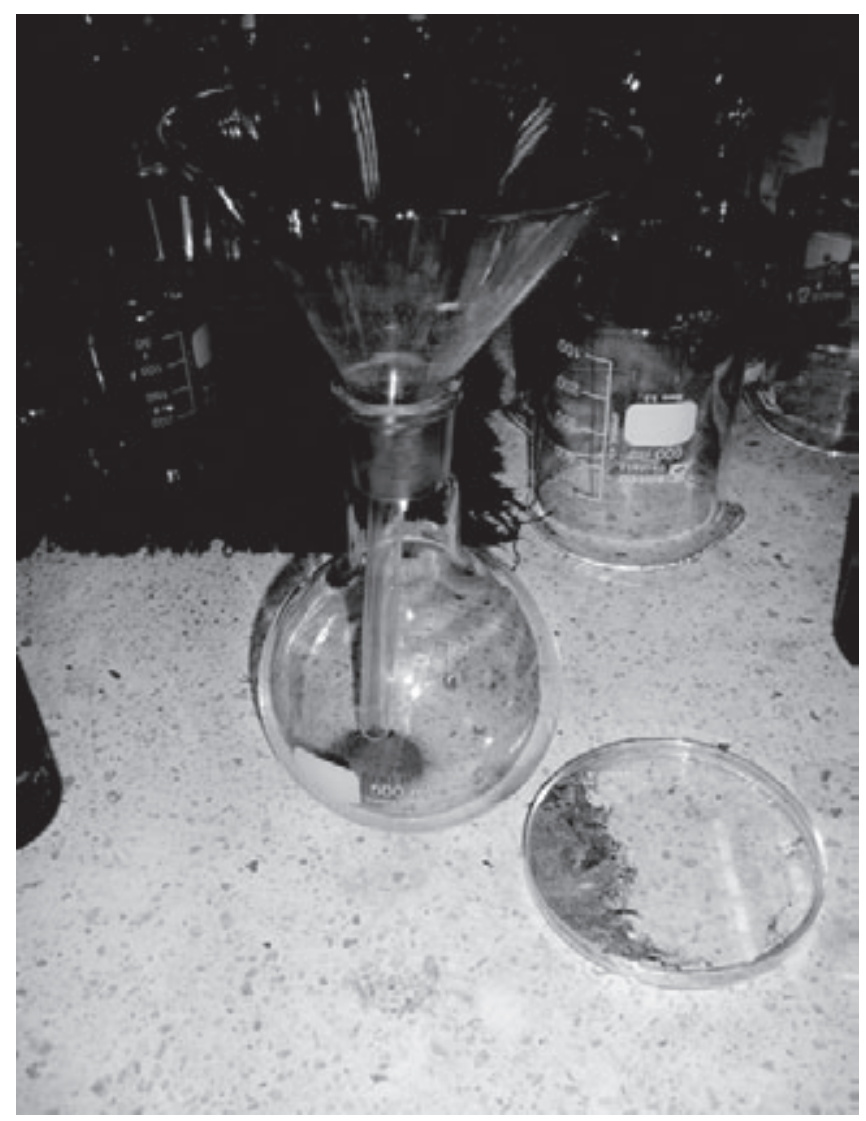

Figura 2. Uso de tierra de diatomeas para decolorar extracto A. 
Las saponinas que cristalizaron de $\mathrm{C}$, tenían la apariencia de gruesos cristales o grumos, de color pardo oscuro, sin olor característico y en concentraciones similares a las utilizadas en la prueba hemolítica; una fuerte positividad se manifestó en la prueba de espuma y en las pruebas para azúcares antrona y molish, en la cuales el producto formado fue azul verdoso y violeta, respectivamente.

\section{OBTENCIÓN DE SAPOGENINAS}

Posterior a la hidrólisis el producto obtenido presentó una coloración amarilla clara y olor dulce característico. Al concentrar el extracto, un sólido de color amarillo claro precipitó (C1 C2 y C3, Figura 4). Lo mismo se realizó con B2 y B3.

El porcentaje total de sapogeninas obtenidas, calculado en forma proporcional a la cuantía de material inicial procesado $(4.95,3.31$ y $6.96 \%)$, fue muy similar al rendimiento obtenido cuando se partió directamente de éstos para obtener los crudos de saponinas (rendimiento $0.085,0.073$ y $0.102 \%$ ) para la primera $(\mathrm{C} 1)$, segunda $(\mathrm{C} 2)$ y tercera procedencia $(\mathrm{C} 3)$ respectivamente.

\section{PURIFICACIÓN DE LAS FRACCIONES C1, C2 Y C3}

Se preparó una cámara de cromatografía con el sistema de solventes cloroformo: acetato de etilo: metanol (3:2:1), que fue el mejor sistema de solventes para las placas W y X, dejando saturar por $30 \mathrm{mi}-$ nutos. Para la placa Y, se utilizaron dos solventes cloroformo: acetato de etilo (1:3). Sobre una placa de cromatografía sílica gel 60 con soporte de aluminio, se realizaron siembras de aproximadamente $20 \mu \mathrm{L}$. De igual manera se aplicaron los patrones de hecogenina y tigogenina sobre la placa. Después de correr la placa se retiró y secó para su detección. Ésta se reveló con un aspersor (Pirex), que contenía la mezcla ácido sulfúrico-vainillina y colocada en una estufa a $110^{\circ} \mathrm{C}$, detectando así tres manchas, dos de color amarillo y una de color café.

Se corrieron las mismas placas para las fracciones C2A y C3A, mostrando las más representativas a continuación, donde se detectó que a pesar de usar la misma proporción de solventes para la placa $\mathrm{X}$ de la fracción (C2A), ésta se diferenció en el Rf (0.58) de la primera placa, donde su Rf (0.45) fue similar al de la fracción $\mathrm{C} 1 \mathrm{~A}$, razón por la cual se decidió correr la placa $\mathrm{Y}$ de la fracción C3A con dos de los solventes ya mencionados, mostrándonos un $\mathrm{Rf}(0.7)$ muy cercano al patrón de tigogenina, a pesar de que la coloración de la mancha siguió siendo amarilla.

Las fracciones puras obtenidas de $\mathrm{C} 1 \mathrm{~A}, \mathrm{C} 2 \mathrm{~A}$ y C3A se identificaron como cristales blancos amorfos con punto de fusión entre $253-269{ }^{\circ} \mathrm{C}$ (tomados en fusiometro MEL-TEMP), pruebas positivas para sapogeninas esteroidales, solubles en cloroformo de coloración característica en placa con vainillinaácido sulfúrico, Rf similar al patrón de hecogenina (placas W y X) y tigogenina (placa Y), por lo que puede haber una sustancia en común, que puede ser hecogenina o tigogenina, para las tres fracciones.

\section{MÉTODOS ESPECTROSCÓPICOS}

La elucidación estructural de la fracción C1A mostró en el espectro IR (Figura 3) una banda ancha y puntiaguda, entre $3.527-3.266 \mathrm{~cm}^{-1}$, característica del grupo Он. Entre 1.708-1.663 $\mathrm{cm}^{-1}$, presentó dos bandas de hombro ancho que indicaron la presencia de un grupo carbonilo. Entre 1.075-1.008 $\mathrm{cm}^{-1}$ se observaron varias bandas características de enlaces C-O simples y finalmente, corroborando el análisis cromatográfico previo, la presencia de cuatro bandas características de sapogeninas esteroidales, debidas a la presencia del anillo F dentro de la aglicona. Estas últimas nos dieron la estereoquímica del C25 dentro de la molécula (Martínez 2001), donde la banda $900 \mathrm{~cm}^{-1}$ es más intensa que la última de ellas, $919 \mathrm{~cm}^{-1}$.

De acuerdo al análisis IR se concluyó que se trata de una sapogenina esteroidal con la presencia de un grupo carbonilo, enlaces $\mathrm{C}-\mathrm{O}$ simples, grupos hidroxilos y grupos metilos geminales.

En el espectro ${ }^{1} \mathrm{H}-\mathrm{RMN}$ de $(\mathrm{C} 1 \mathrm{~A})$, registrado a 400 MHZ, la mayoría de las señales se encontraron en el rango de $\delta=0.5-2.5 \mathrm{ppm}$ (Figura 4), una alrededor 


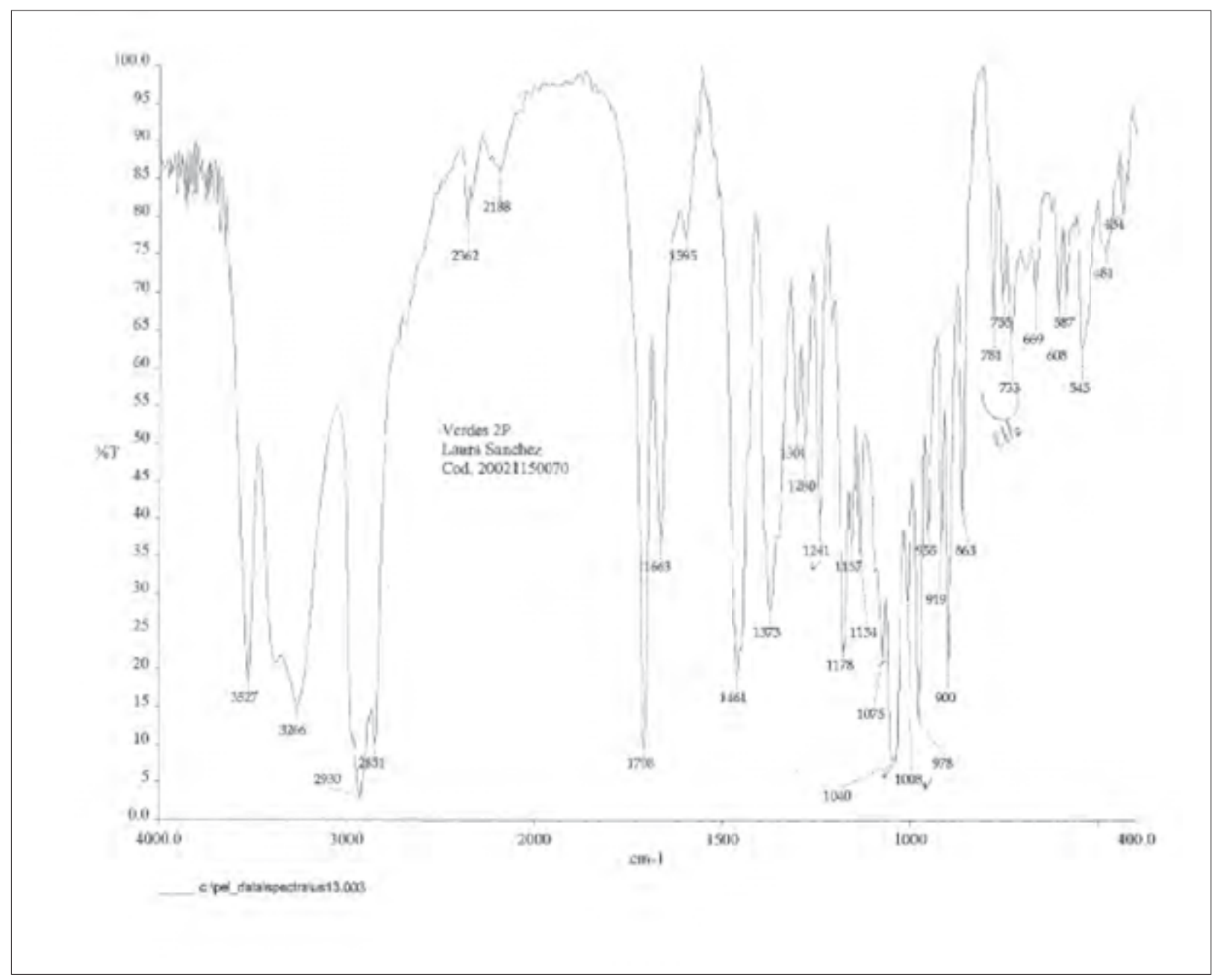

Figura 3. Espectro IR de las fracciones C1A, C2A y C3A (anexo D).

de $4.4 \mathrm{ppm}$ y tres alrededor de $3.5 \mathrm{ppm}$. De estas últimas la primera correspondió a hidrógenos asociados a carbonos electronegativos como el oxígeno que puede tratarse de enlaces $\mathrm{C}-\mathrm{O}$ simples y las segundas a carbonos asociados a grupos $\mathrm{OH}$.

El espectro mostró múltiples señales solapadas entre 1.2 y $1.7 \mathrm{ppm}$, que no permitieron distinguir la cantidad de hidrógenos posibles en la molécula. Sólo se pudo deducir que correspondía a protones $\mathrm{CH}$ у $\mathrm{CH} 2$. Los protones de grupos metilo se observaron entre 0.7 y $1.06 \mathrm{ppm}$.

El espectro ${ }^{13} \mathrm{C}-\mathrm{RMN}$ mostró 54 señales con las cuales, según los análisis del trabajo previo, podríamos esperar que el aglicón fuera un esqueleto esteroidal (27 átomos de carbono), por lo cual la sustancia corresponde a una mezcla de dos aglicones esteroidales. Esto es apoyado por el experimento DEPT 135 que presenta $18 \mathrm{CH}$ (desplazamientos mayores a $35 \mathrm{ppm}$, parte positiva del espectro), 7 cH3 (desplazamientos menores a 35 ppm, parte positiva del espectro, $20 \mathrm{CH} 2$ (parte negativa del espectro) y 6 señales que no se encuentran en el DEPT $135(\delta=35.544,36.061,55.078$, 40.532, 102.9, 213.59), pero sí en ${ }^{13} \mathrm{C}-\mathrm{RMN}$ : son los carbonos cuaternarios que fueron confirmados en el experimento HMBC (donde 102.9 ppm se encuentra solapada).

Para identificar las estructuras de los aglicones esteroidales utilizamos el experimento HMBC, que es el más adecuado para obtener la asignación de los anillos y la de los 20 grupos $\mathrm{CH}_{2}$. De esta forma la 

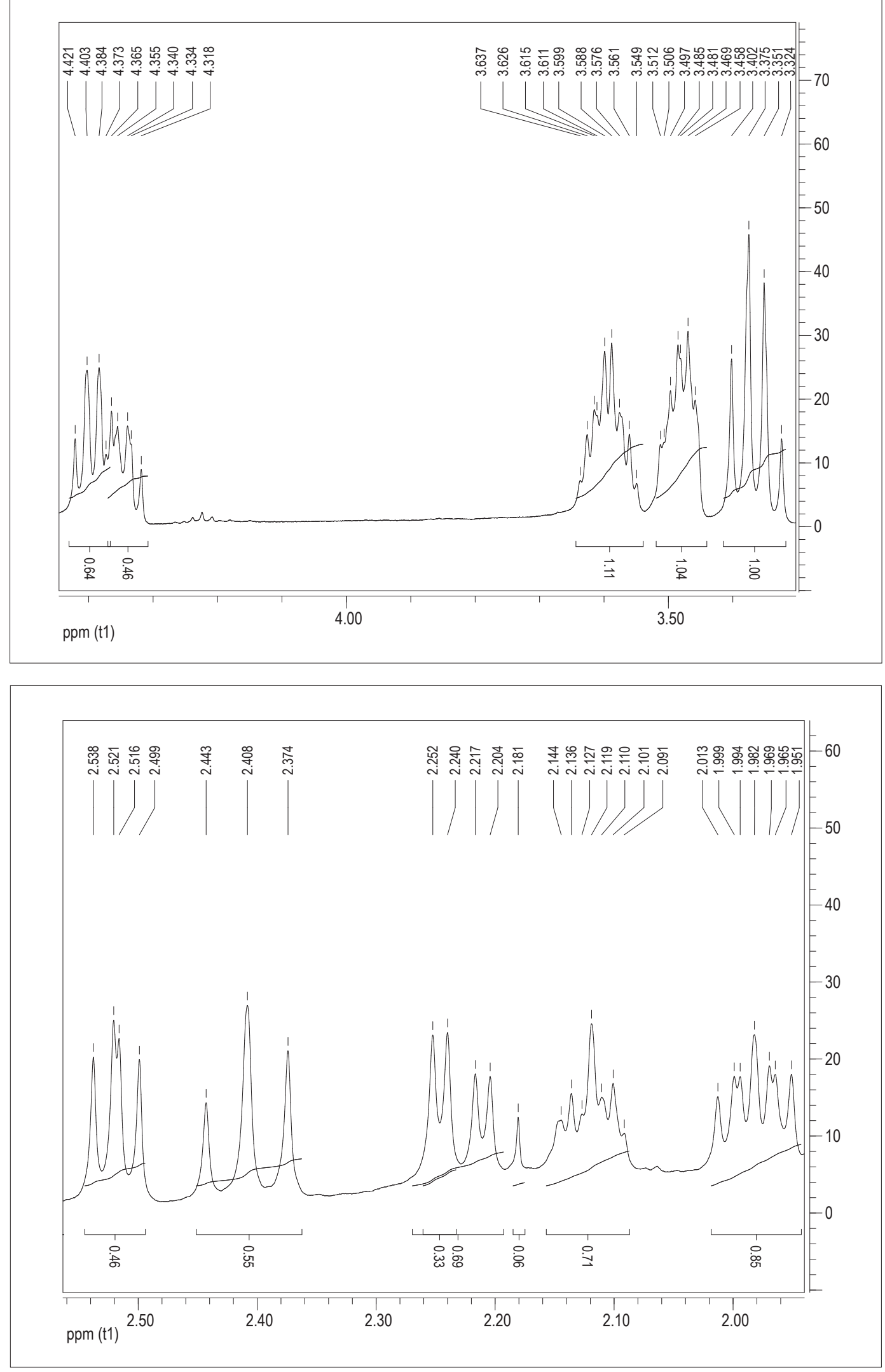

Figura 4. Espectro ${ }^{1} \mathrm{H}-\mathrm{RMN}$ de la fracción $\mathrm{C} 1 \mathrm{~A}$ (anexos E1 yE2). 


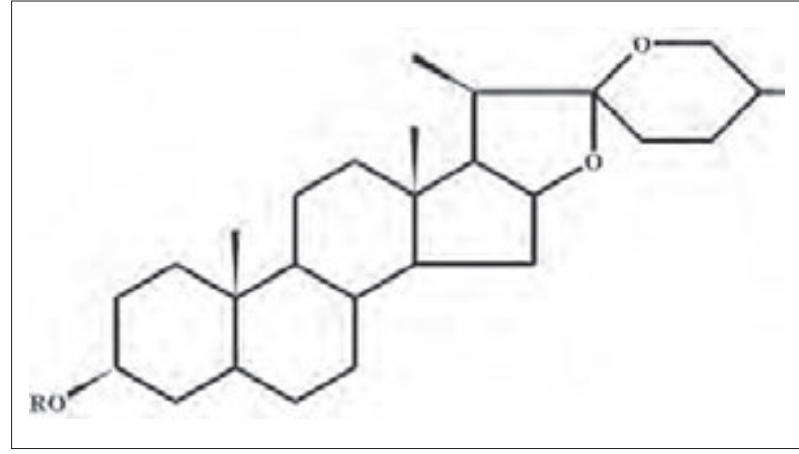

Figura 5. Estructura de la sustancia a (25 R) 12-oxa-espirostan3-ol (derecha).

correlación a 2 y 3 ligaduras hizo posible la asignación de la mayoría de los carbonos del esqueleto.

El desplazamiento elevado de C16 $(\delta=79.162$ y 80.816 para a y b respectivamente), que es la señal más cercana al grupo carbonilo $\mathrm{C} 12 \mathrm{a}$ en ${ }^{13} \mathrm{C}-\mathrm{RMN}$ a campos bajos del espectro, nos permite asignar a éste la unión con un átomo de oxigeno, que de acuerdo al análisis IR debe ser un enlace C-O simple (1.075$\left.1.008 \mathrm{~cm}^{-1}\right)$ y que muestra en ${ }^{1} \mathrm{H}-\mathrm{RMN}(\delta=4.32$ y 4.4 $\mathrm{J}=6.4$ y $7.2 \mathrm{~Hz}$ para a y $\mathrm{b}$ ) un doble doblete y un cuarteto, respectivamente (Figuras 5 y 6 ).

Los fragmentos I y II se interconectan a través de C13a y C14a, como mostró el punto de cruce en HMBC, lo mismo sucedió para la estructura b. La

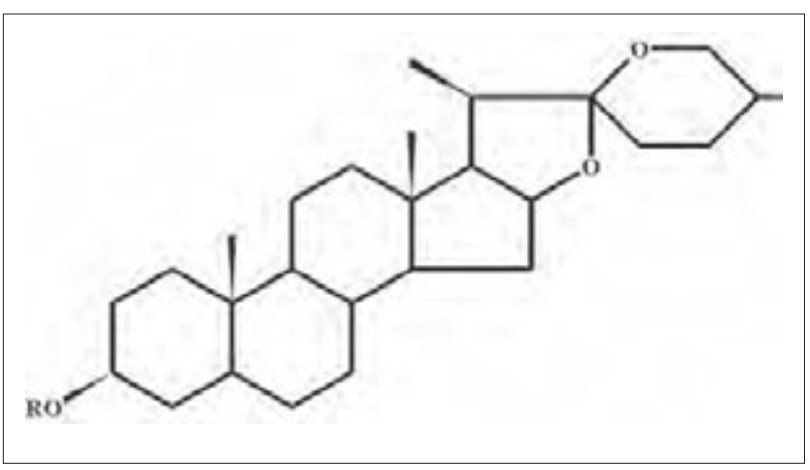

Figura 6. Estructura de la sustancia b (25 R) espirostan-3-ol (izquierda).

estereoquímica de $\mathrm{C} 25$ ( $\delta=30.148$ y 30.255 para a y $\mathrm{b}$ respectivamente), según el análisis IR, corresponde a R y se corrobora por el desplazamiento químico de los protones de los metilos $21(\delta=1.06$ y $0.96, \mathrm{~J}=$ 7.2 у $6.8 \mathrm{~Hz})$ у $27(\delta=0.8$ y $0.75, \mathrm{~J}=6.4$ у $6.2 \mathrm{~Hz})$, que resuenan dobletes entre 0.75-1.06 ppm, según la literatura (Martínez 2001).

\section{CÁLCULO DE RENDIMIENTO APROXIMADO DE SAPOGENINAS ESTEROIDALES}

La procedencia que presentó el mayor rendimiento tanto en el crudo de sapogeninas (6.96, Figura 7), como en la fracción purificada C3A (0.102, Figura 8), fue la del Alto de San Miguel. En las otras dos

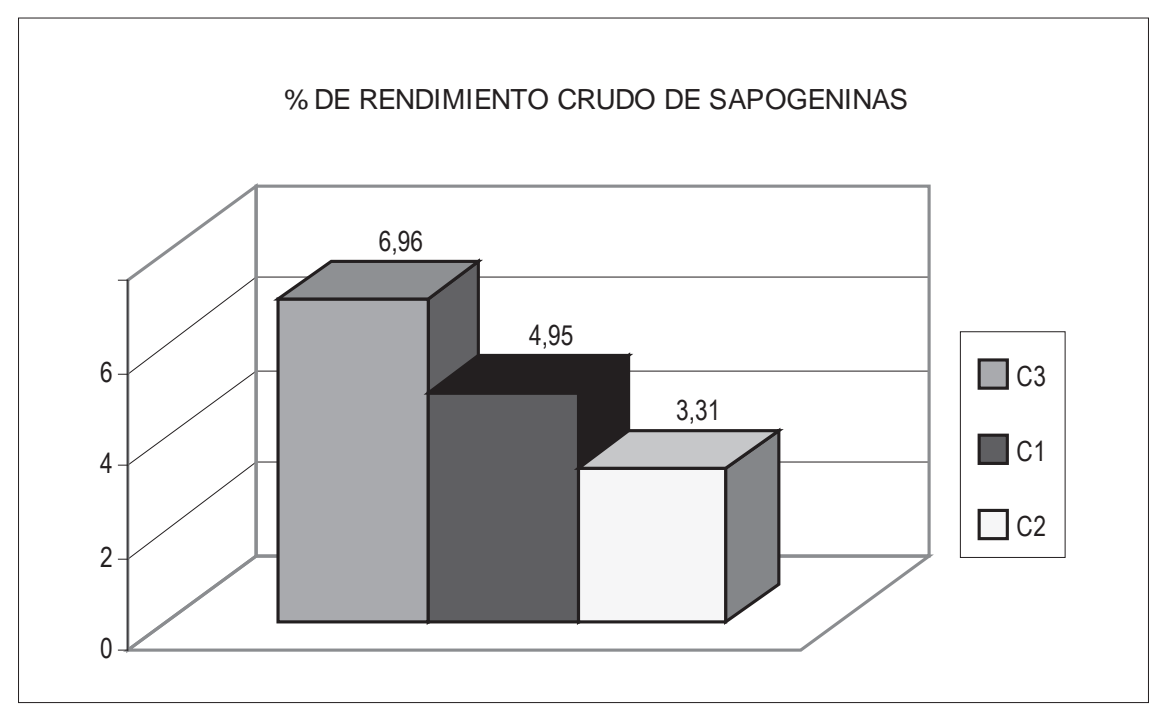

Figura 7. Porcentaje de rendimiento de crudo de sapogeninas. 


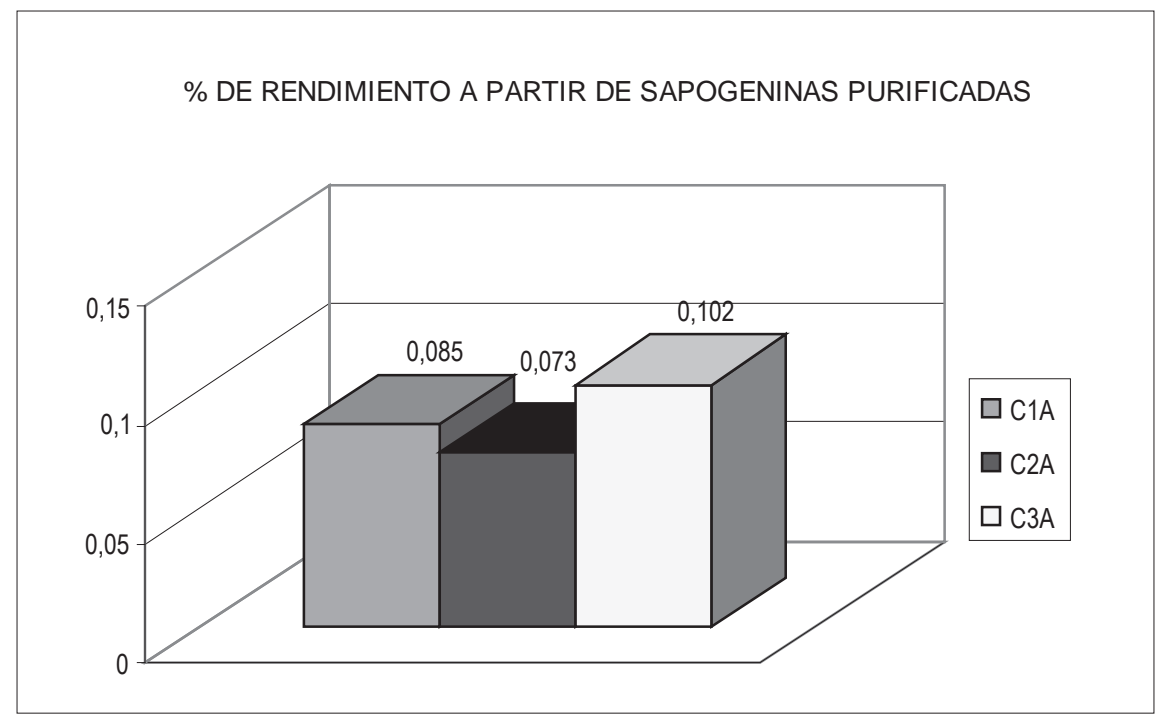

Figura 8. Rendimiento de sapogeninas purificadas.

procedencias no se encontraron diferencias significativas entre los porcentajes de rendimiento de las primera y segunda sapogeninas purificadas $(0.085$ 0.073 respectivamente), pero sí de la tercera procedencia (0.102).

Los rendimientos a partir del material inicial para el crudo de sapogeninas esteroidales (Figura 7) y después del proceso de purificación (Figura 8) estuvieron entre 3.31 y $6.96 \%$ y 0.073 y 0.102 por mil respectivamente.

\section{DISCUSIÓN}

\section{COMPARACIÓN DE ACUERDO CON LOS ESTADOS DE MADURACIÓN}

El mejor estado de maduración correspondió al verde pues, de acuerdo al tamizaje fitoquímico, en éste se reveló la presencia de triterpenos, esteroles y saponinas, como se mostró en la cromatografía en capa delgada, que de acuerdo a la bibliografía (Bilbao 1997) son coloraciones establecidas para esteroides y 3 zonas hemolizadas, que corresponden a un resultado efectivo para saponinas (Bilbao 1997). Se descarta cualquier presencia de alcaloides en la planta, de lo cual se concluye que las sapogeninas presentes en ella son esferoidales y no alcaloidales.

\section{SAPOGENINAS ESTEROIDALES}

Las sapogeninas se hidrolizaron dos veces con $\mathrm{H}_{2} \mathrm{SO}_{4}$, hasta que la prueba para saponinas fue negativa, lo que nos permitió deducir que hay gran presencia de polisacáridos, que no son fácilmente hidrolizados por efecto del ácido. Los productos obtenidos presentaron una coloración amarilla clara, por lo cual fue necesario un proceso de purificación por medio de lavados con cloroformo.

De los colores característicos obtenidos en CCD y de acuerdo a la bibliografía citada (Bilbao 1997), la especie sólo presentó sapogeninas esteroidales, lo cual confirmó lo expresado por Fontan (1973), pues no se han encontrado sapogeninas triterpenoidales (coloración violeta característica) en la familia Solanaceae.

\section{CÁLCULO DE RENDIMIENTO DE LAS SAPOGENINAS ESTEROIDALES}

El porcentaje total de sapogeninas obtenidas, calculado en forma proporcional a la cuantía de material inicial procesado $(4.95,3.31$ y $6.96 \%)$, fue muy similar al rendimiento obtenido cuando se partió directamente de éstos para obtener los crudos de saponinas (rendimiento 0.085, 0.073 y $0.102 \%$ ) para la primera (C1), segunda (C2) y 
tercera procedencia $(\mathrm{C} 3)$ respectivamente. Esto indica una presencia proporcional tanto de saponinas como de sapogeninas para las tres procedencias.

\section{MÉTODOS}

\section{ESPECTROSCÓPICOS}

De acuerdo al análisis IR se concluyó que se trata de una sapogenina esteroidal con la presencia de un grupo carbonilo, enlaces $\mathrm{C}-\mathrm{O}$ simples, grupos hidroxilos y grupos metilos geminales.

El desplazamiento elevado de $\mathrm{C} 16(\delta=79.162$ y 80.816 para a y b respectivamente), que es la señal más cercana al grupo carbonilo $\mathrm{C} 12 \mathrm{a}$ en ${ }^{13} \mathrm{C}-\mathrm{RMN}$ a campos bajos del espectro, nos permitió asignar a éste la unión con un átomo de oxígeno, que de acuerdo al análisis IR, debe ser un enlace C-O simple $\left(1.075-1.008 \mathrm{~cm}^{-1}\right)$ y que muestra en ${ }^{1} \mathrm{H}-\mathrm{RMN}$ $(\delta=4.32$ y $4.4, \mathrm{~J}=6.4$ y $7.2 \mathrm{~Hz}$ para a y b) un doble doblete y un cuarteto, respectivamente.

Los fragmentos I y II se interconectan a través de C13a y C14a, como mostró el punto de cruce en HMBC, lo mismo sucedió para la estructura b. La estereoquímica de $\mathrm{C} 25$ ( $\delta=30.148$ y 30.255 para a y $\mathrm{b}$ respectivamente), según el análisis IR, correspondió a $\mathrm{R}$ y se corroboró por el desplazamiento químico de los protones de los metilos $21(\delta=1.06$ у $0.96, \mathrm{~J}=7.2$ у $6.8 \mathrm{~Hz})$ у $27(\delta=0.8$ у $0.75, \mathrm{~J}=$ 6.4 y $6.2 \mathrm{~Hz}$ ) que resuenan dobletes entre 0.75-1.06 ppm, según la literatura (Martínez 2001).

\section{CÁLCULO DE RENDIMIENTO APROXIMADO DE SAPOGENINAS ESTEROIDALES}

El rendimiento fue superior al reportado en la literatura revisada (Schäfer et al. 2006) para la realización de este proyecto, en ella se obtuvieron rendimientos entre 0.003 y $0.027 \%$ en alto estado de pureza para cuatro diferentes plantas de este género. Lo cual expresó la confiabilidad del método empleado y la semejanza en contenido de los diversos procesos utilizados, así como la viabilidad que tendría el fruto en el mercado para la obtención de diferentes productos farmacológicos como hormonas sexuales (progesterona), hormonas corticoides (prednisolona y cortisona) y hormonas anabólicas (stanozolol y methadienona, Pradilla et al. 2006).

\section{CONCLUSIONES}

Teniendo en cuenta el tamizaje fitoquímico realizado y las pruebas cualitativas para los metabolitos de interés, los siguientes son los grupos fitoquímicos encontrados para la especie: carotenoides, ácidos grasos, cumarinas, taninos, saponinas, esteroides y triterpenos y polisacáridos. Estos metabolitos, así como los de sapogeninas esteroidales, se encontraron en el estado de maduración verde, razón por la cual son los más apropiados para la extracción de dichas sustancias, siendo la mejor procedencia el Alto de San Miguel. Sin embargo las tres procedencias mostraron presencia de las sapogeninas esteroidales, punto de fusión entre $253-269^{\circ} \mathrm{C}$, solubilidad alta en cloroformo, coloración característica en placa para sapogeninas esteroidales $y$, de acuerdo a los análisis espectroscópicos, estructuras análogas a hecogenina y tigogenina.

Los rendimientos aproximados de sapogeninas en alto estado de purificación obtenidos para las tres procedencias se encontraron entre 0.073 y $0.102 \%$ en peso. La tercera procedencia, el Alto de San Miguel, fue la que presentó mayor rendimiento, no sólo a partir del crudo de sapogeninas, sino también después de la purificación de los cristales obtenidos. Lo cual se atribuyó principalmente al sitio donde se encontraron los frutos, pues allí estaban al cuidado de la población de la zona, donde ellos son utilizados para elaborar luladas, además de las condiciones de altura, pues presentaban condiciones entre 2.400 y $2.460 \mathrm{msnm}$ y temperaturas más bajas con respecto a las otras procedencias.

El porcentaje determinado es mayor en comparación a estudios paralelos con especies de la misma familia utilizando el mismo método, lo cual hace del fruto de Solanum quitoense var. Septentrionale, dentro del marco de especies andinas y de páramo, una especie de interés para la extracción de sapogeninas esteroidales.

La relevancia de encontrar resultados tan positivos en esta investigación hace que un campo 
disciplinar como la fitoquímica nos muestre nuevas opciones para buscar especies químicas que son de gran importancia para la salud, la alimentación y las nuevas tecnologías, obtenidas no sólo de la explotación primaria de las plantas sino de otros recursos potencialmente mejores.

\section{AGRADECIMIENTOS}

Esta investigación se realizó con el apoyo financiero y la dotación de algunos de los materiales biológicos del Jardín Botánico José Celestino Mutis. Al profesor Jairo Silva por su invaluable labor y compromiso con el presente trabajo, su constancia y confianza.

\section{REFERENCIAS BIBLIOGRÁFICAS}

Bilbao, M. 1997. Análisis fitoquímico preliminar. Universidad del Quindío. Editorial Norma, Armenia. Pg. 185.

Ciulei, I. 1982. Metodología de análisis de productos vegetales con fines farmacológicos. Facultad de Farmacia, Bucarest, Rumania.

Fanor, R. C. 2001. Cálculo de la humedad y otros factores en la extracción de compuestos activos.
Industrialización de metabolitos secundarios 3: 28-31.

Fontan, J. I. 1973. Las saponinas y la botánica. Instituto Español de Fisiología y Bioquímica. McGraw-Hill, Madrid. Pg. 367.

Ibáñez, T. 1981. Industrialización de las plantas medicinales de Colombia. Universidad de Santiago. Revista Creces 25: 21-31.

Martínez, M. A. 2001. Saponinas esteroides. Revista UDEA 3: 2-19.-

Pradilla, A., H. Laverde \& J. C. Pabón. 2006. Prospectiva tecnológica de la cadena productiva agroindustrial del fique en el departamento del Santander. CBE y Colciencias. Págs. 25-32.

Sanabria, A. 1983. Análisis fitoquímico preliminar, metodología y su aplicación en la evaluación de 40 plantas de la familia compositae. Universidad Nacional de Colombia. Fitochem: 21-23.

Schafer, G., S. Urrego \& G. Palacio. 2006. Extracción, purificación e identificación de sapogeninas de plantas del género Solanum. Eafit, Revista Ingeniería y Ciencia 5: 2-10. 